\title{
Características da utilização de serviços de Atenção Básica à Saúde nas regiões Sul e Nordeste do Brasil: diferenças por modelo de atenção
}

\author{
Characteristics of primary healthcare service use in the southern \\ and northeastern regions of Brazil: differences by care model
}

\author{
Elaine Tomasi ${ }^{1}$ \\ Luiz Augusto Facchini ${ }^{2}$ \\ Elaine Thumé ${ }^{3}$ \\ Roberto Xavier Piccini ${ }^{2}$ \\ Alessander Osorio ${ }^{2}$ \\ Denise Silva da Silveira ${ }^{2}$ \\ Fernando Vinholes Siqueira ${ }^{4}$ \\ Vanessa Andina Teixeira ${ }^{1}$ \\ Aliteia Santiago Dilélio ${ }^{3}$ \\ Maria de Fátima Santos Maia ${ }^{5}$
}

${ }^{1}$ Universidade Católica de Pelotas. Av. Fernando Osório 1586, Três Vendas. 96055-000 Pelotas RS. tomasiet@uol.com.br

${ }^{2}$ Departamento de Medicina

Social, Faculdade de

Medicina, Universidade

Católica de Pelotas

${ }^{3}$ Faculdade de Enfermagem

e Obstetrícia, Universidade

Federal de Pelotas

4 Curso de Terapia

Ocupacional, Universidade

Federal de Pelotas

${ }^{5}$ Universidade Federal do

Rio Grande

\begin{abstract}
In view of the emphasis on primary care for the health system and the consolidation of Family Healthcare Strategy, it is important that information on attended demand be updated to assist in administration of the Unified Health System (UHS). The study compared the profile of attended demand in basic healthcare units (BHU) of two care models (traditional and family health) in 240 BHU of seven states of the South and the Northeast. Collected on a prepared form, all patients attended in a single day were processed with the PACOTAPS application. 26,019 patients were attended, 52\% in the South and 48\% in the Northeast; one third in Traditional BHU and $67 \%$ in BHU of FHP. The highest proportion of patients attended was females aged between 15 and 49 (36\%), with significant differences between the models, being higher in BHU of FHP. The second highest proportion was people aged 50 or older (30\%), significantly higher in Traditional BHU. The most commonly registered procedures were basic nursing cases (33\%), with higher proportion in Traditional BHCU. The proportion of medical appointments was 22\%, double in Traditional BHCU. The profile of the demand reflected the differences between the ongoing care models in the country and may provide the organization of work processes in basic care.
\end{abstract}

Key words Primary health care, Outpatient demand, Family health, UHS
Resumo Considerando o destaque da atenção básica para o sistema de saúde e a consolidação da Estratégia da Saúde da Família, é importante que as informações sobre a demanda atendida sejam atualizadas para apoiar a gestão do SUS. O estudo comparou o perfil da demanda atendida em unidades básicas de saúde (UBS) de dois modelos de atenção (tradicional e saúde da família [PSF]) em 240 UBS de sete estados do Sul e Nordeste. Coletados em formulário próprio, todos os atendimentos de um dia de trabalho foram processados com o aplicativo PACOTAPS. Foram registrados 26.019 atendimentos, 52\% no Sul e 48\% no Nordeste; um terço em UBS Tradicionais e $67 \%$ em UBS do PSF. A maior proporção de atendimentos foi para mulheres entre 15 e 49 anos (36\%), com diferenças significativas entre os modelos, sendo maior nas UBS do PSF. A segunda maior proporção foi de pessoas com 50 anos ou mais de idade (30\%), significativamente maior nas UBS Tradicionais. Os procedimentos mais registrados foram os atendimentos básicos de enfermagem (33\%), com maior proporção nas UBS Tradicionais. A proporção de consultas médicas foi de $22 \%$, sendo duas vezes maior nas UBS Tradicionais. O perfil da demanda refletiu as diferenças entre os modelos de atenção no país e pode subsidiar a organização dos processos de trabalho em atenção básica.

Palavras-chave Atenção básica à saúde, Demanda ambulatorial, Saúde da família, SUS 


\section{Introdução}

Os sistemas de saúde baseados no fortalecimento da atenção básica estão organizados de modo a atender a maior parte dos problemas de saúde e a enfatizar ações de promoção da saúde e de prevenção dos agravos. Sua utilização é resultante da interação do comportamento do indivíduo que procura cuidados, do profissional que o conduz dentro do sistema de saúde e dos recursos diagnósticos e terapêuticos disponibilizados. Entre seus determinantes, podem ser destacados aqueles relacionados às necessidades de saúde, aos usuários e às características da oferta dos serviços ${ }^{1-3}$.

A demanda por serviços de saúde pode ser entendida como o desejo e/ou a habilidade do indivíduo em procurar, utilizar e, em algumas circunstâncias, pagar pelos serviços recebidos. Em termos operacionais, pode ser subdividida em demanda potencial, que é a percepção de necessidade do indivíduo e sua decisão de buscar um atendimento e em demanda expressa ou atendida, que é a utilização propriamente dita ${ }^{4}$.

Ao buscar o serviço para ações profiláticas, de monitoramento e para problemas agudos ou crônicos, o acesso dependerá, em primeiro lugar, de sua disponibilidade, e em segundo, da capacidade dos sujeitos de transitarem pelo sistema de saúde, inclusive em função de sua situação econômica ${ }^{4}$.

A continuidade da utilização passa a incluir, além das características da demanda, a decisão médica com base no diagnóstico sobre o problema do usuário e dos recursos disponíveis para tratá-lo, afetando o número de vezes que o indivíduo precisa retornar ao serviço, após o contato inicial ${ }^{5}$.

Estudo americano com 1000 indivíduos (homens, mulheres e crianças), sobre o padrão de acesso e de utilização de serviços durante o período de um mês, verificou que 800 pessoas se referiram a um "sintoma" (80\%), 327 "pensaram" em consultar um médico (32,7\%), 217 consultaram um médico (21,7\%), 113 consultaram um médico da atenção primária em saúde (11,3\%), 104 consultaram especialistas (10,4\%), 65 consultaram um especialista para cuidado médico complementar ou alternativo $(6,5 \%), 21$ consultaram um ambulatório especializado de hospital (2,1\%), 14 receberam cuidado de profissional de saúde em casa (1,4\%), 13 foram atendidos em pronto-socorro $(1,3 \%), 8$ foram hospitalizados $(0,8 \%)$ e um foi encaminhado para hospital universitário $(0,1 \%)^{6}$.

No Brasil, a Pesquisa Nacional por Amostra de Domicílios ${ }^{7}$ sobre o padrão de acesso e de utilização de serviços, publicada em 2003, referiu que, do total de entrevistados, 14\% relataram ter procurado um serviço de saúde nos 15 dias que antecederam a entrevista, e destes $96 \%$ foram atendidos, sendo 59\% em serviços do Sistema Único de Saúde (SUS) e 41\% no sistema privado.

Se o conhecimento sobre o padrão de utilização e as características dos usuários dos serviços de saúde é fundamental para o planejamento do sistema de saúde como um todo, esta importância aumenta em relação à atenção básica, por constituir-se em porta de entrada do sistema público e por responder por cerca de $30 \%$ dos atendimentos em saúde no Brasil ${ }^{8}$.

Diferentes estudos têm descrito a utilização de serviços primários em saúde e seus principais fatores associados. Os grupos extremos de idade (crianças e idosos) utilizam mais os serviços e o número de consultas aumenta com a idade. As mulheres consultam mais do que os homens, mesmo após ajustes para necessidades em saúde. $\mathrm{O}$ diferencial se explica, em parte, por um interesse maior do gênero feminino pela sua condição de saúde $e^{9,10}$. A escolaridade e a situação socioeconômica são outros fatores considerados importantes preditores de utilização de serviços de saúde, uma vez que se associam a um maior conhecimento e à adoção de comportamentos mais saudáveis ${ }^{11-13}$.

Sabe-se que a opção mais adequada para conhecer o perfil de morbidade de uma população é a realização de inquéritos de base populacional $^{3,14,15}$. Entretanto, isto nem sempre é possível, exigindo recursos humanos e financeiros de relativa magnitude. Uma alternativa bastante utilizada constitui-se na realização de estudos de demanda que podem fornecer informações sobre o perfil de morbidade e os motivos da busca por atendimento em uma unidade de saúde, como por exemplo, procedimentos preventivos e curativos, sejam eles vinculados a ações de saúde programáticas ou não ${ }^{15,16}$.

A proposta de atenção primária em saúde, expressa na Conferência de Alma-Ata, representou uma inovação conceitual e tecnológica na visão sobre os sistemas de saúde no mundo, ao defender os princípios de integralidade, qualidade, equidade e participação social em saúde. Estes princípios seriam alcançados através de uma rede descentralizada de serviços de saúde, capaz de acolher e resolver boa parte dos problemas de saúde de indivíduos e comunidades, priorizando grupos sociais mais vulneráveis ${ }^{17}$.

A partir de 1994, com a implantação progressiva da Estratégia da Saúde da Família, a atenção básica à saúde no Brasil ${ }^{18}$ se fortalece anco- 
rada nos princípios de integralidade e hierarquização da atenção, territorialização, cadastramento da população e equipe multiprofissional ${ }^{19,20}$.

Reforçada com o crescimento da cobertura da Estratégia da Saúde da Família ${ }^{21-23,3}$ e com o destaque recebido no último Relatório Mundial de Saúde ${ }^{24}$, a atenção básica poderia se qualificar com a atualização de informações sobre a demanda atendida, de forma a permitir sua efetiva contribuição à gestão do sistema de saúde.

Entretanto, os estudos de demanda costumam se restringir à avaliação de um serviço em particular ou de um território mais delimitado, havendo carência de estudos de maior abrangência capazes de revelar diferenças entre contextos macro-regionais e de modelos de atenção.

O objetivo deste artigo foi comparar o perfil da demanda atendida em unidades básicas de saúde (UBS) de dois modelos de atenção (tradicional e saúde da família) em sete estados brasileiros das regiões Sul e Nordeste.

\section{Métodos}

Os dados analisados integraram o estudo de avaliação do Programa de Saúde da Família (PSF), através do Projeto de Expansão e Consolidação da Saúde da Família (PROESF) ${ }^{25}$. A Universidade Federal de Pelotas (UFPel) foi responsável pelo Estudo de Linha de Base (ELB) do PROESF em 41 municípios com mais de cem mil habitantes das regiões Sul (Rio Grande do Sul e Santa Catarina) e Nordeste (Alagoas, Paraíba, Pernambuco, Piauí e Rio Grande do Norte) ${ }^{3}$.

O delineamento foi transversal a partir de unidades básicas de saúde e sua seleção seguiu inicialmente um critério de estratificação de acordo com o modelo de atenção (PSF e Tradicional). Em oficinas macrorregionais ${ }^{26}$, representantes da gestão municipal da saúde apresentaram listas das UBS em cada município, por localização (urbana e rural), modelo de atenção e área física. O total de UBS era de 626 no Sul e de 855 no Nordeste. Buscando caracterizar entre $10 \%$ e $20 \%$ de UBS urbanas e respeitando a proporcionalidade dos modelos de atenção em relação à capacidade instalada da rede básica em cada município, chegouse a uma amostra de 120 UBS por região. Durante as oficinas, as 240 UBS foram selecionadas aleatoriamente, na razão de duas com PSF para uma Tradicional. Esta razão foi definida em função do objetivo do ELB que era comparar o desempenho da atenção básica em UBS pré e pós PROESF versus atenção tradicional.
O formulário utilizado na coleta de dados continha todas as informações da Ficha de Atendimento Ambulatorial (FAA) do Sistema de Informações Ambulatoriais do Sistema Único de Saúde (SIA/SUS) - documento rotineiro nas UBS para efeitos gerenciais e contábeis. Foi solicitado que os profissionais da UBS incluindo os Agentes Comunitários de Saúde (ACS) registrassem todos os atendimentos realizados durante um dia de trabalho, sob a orientação de uma equipe de supervisores capacitados.

A Tabela de Procedimentos do SIA-SUS foi utilizada para identificação dos procedimentos. Após codificação das variáveis e revisão, os formulários foram digitados no software PACOTAPS $^{27}$, capaz de informar sobre a qualidade do registro das atividades, além de produzir análises sobre o perfil demográfico e epidemiológico da demanda das UBS. O software foi distribuído aos municípios durante as Oficinas de Capacitação e os representantes das secretarias municipais de saúde (SMS) e das UBS foram capacitados para sua utilização ${ }^{26,28}$. Este aplicativo também pode ser obtido na página do Projeto na Internet (www.epidemio-ufpel.org.br/proesf/index.htm).

As análises finais foram feitas através de pacote estatístico, após conversão dos bancos de dados. As características selecionadas como desfecho foram os subgrupos de idade e sexo (menores de cinco anos, de cinco a catorze anos, mulheres de 15 a 49 anos, homens de 15 a 49 anos, de 50 a 64 anos e de 65 anos e mais), a escolaridade (analfabetos, os que somente sabem assinar, os com fundamental incompleto, fundamental completo e médio incompleto, médio completo e superior - tanto completo quanto incompleto e fora da idade escolar), os profissionais (enfermeiro, médico, odontólogo, outro nível superior, nível médio e ACS) e os procedimentos (imunização, atendimento básico de enfermagem, visita domiciliar, consulta médica, prevenção em odontologia, dentística e exodontia e outro procedimento de nível superior). Os atendimentos básicos de enfermagem incluíram aferição de peso e altura/estatura, verificação de pressão arterial, realização de curativos e administração de medicamentos. Os outros procedimentos de nível superior incluem consultas e atendimentos específicos realizados por enfermeiros, psicólogos e nutricionistas.

A variável de exposição foi o modelo de atenção das UBS (Tradicional e PSF) e a variável de estratificação foi a região geográfica (Sul e Nordeste), buscando destacar o efeito do contexto regional no exame das associações. 


\section{Resultados}

Foram registrados 26.019 atendimentos realizados por profissionais de 234 UBS (98\% do total de 240 amostradas), em um dia de trabalho, 13.612 (52\%) na região Sul e 12.407 (48\%) na região Nordeste do Brasil. Do total, 8.618 (33\%) aconteceram em 82 UBS Tradicionais e 17.401 (67\%) em 152 UBS do PSF (Figura 1).

O número de atendimentos em cada unidade de saúde variou de 7 a 709, com mediana de 131 atendimentos, sendo 119 nas Tradicionais e 138 nas de PSF. A proporção de registros inferiores a 100 foi de $26 \%$ - 29\% nas Tradicionais e $25 \%$ nas de PSF; entre 100 e 200 atendimentos foram registrados para $56 \%$ das unidades $-52 \%$ nas Tradicionais e $62 \%$ nas de PSF; mais de 200 atendimentos foram registrados para $18 \%$ das UBS 19\% das Tradicionais e 13\% das de PSF.

A Tabela 1 apresenta o perfil da demanda de acordo com o modelo de atenção das UBS. A maior proporção de atendimentos foi para mulheres entre 15 e 49 anos (36\%), com diferenças estatisticamente significativas entre os modelos de atenção, sendo maior nas UBS do PSF $(\mathrm{p}<0,001)$. A segunda maior proporção foi a do grupo de pessoas com 50 anos ou mais de idade (30\%), significativamente maior nas UBS Tradicionais $(\mathrm{p}<0,001)$. A proporção de atendimentos a menores de cinco anos (15\%) foi semelhante em ambos os modelos de atenção. Os idosos representaram $13 \%$ da amostra, e foram significativamente mais atendidos em UBS Tradicionais $(\mathrm{p}<0,001)$. As menores proporções foram das crianças entre cinco e catorze anos (10\%) e homens entre 15 e 49 anos (9\%), mais frequentes em UBS Tradicionais $(\mathrm{p}<0,01)$.

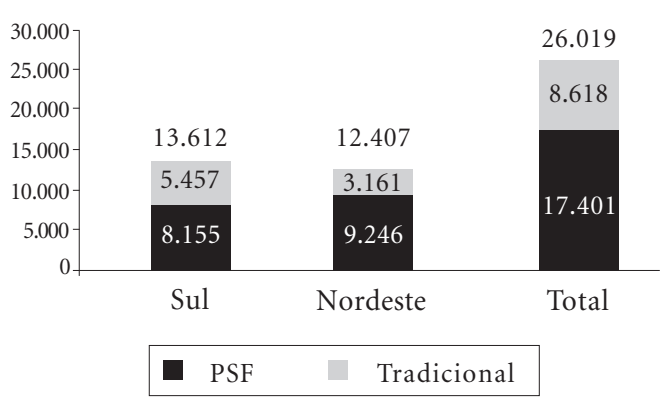

Figura 1. Distribuição da demanda atendida em unidades básicas de saúde. PROESF-UFPEL, 2005.
Com relação à escolaridade foi observado que mais de um quarto das pessoas atendidas era analfabeto ou apenas sabia assinar o nome e esta proporção foi maior nas UBS do PSF $(\mathrm{p}<0,05)$. Cerca de um terço de todos os atendimentos foram de usuários com ensino fundamental incompleto, não havendo diferenças entre os modelos de atenção. $\mathrm{O}$ ensino fundamental completo ou médio incompleto foi registrado para $15 \%$ da amostra, com proporções maiores nas UBS Tradicionais $(\mathrm{p}<0,05)$. Atendimentos de crianças fora da idade escolar representaram $13 \%$ e esta proporção foi maior nas UBS Tradicionais $(\mathrm{p}<0,001)$.

Os procedimentos mais registrados foram os atendimentos básicos de enfermagem (33\%), com maior proporção nas UBS Tradicionais $(\mathrm{p}<0,001)$. As visitas domiciliares responderam por $22 \%$ dos atendimentos, sendo quatro vezes maior nas UBS do PSF do que nas Tradicionais $(\mathrm{p}<0,001)$. A proporção de consultas médicas também foi de $22 \%$, sendo duas vezes mais frequentes nas UBS Tradicionais $(\mathrm{p}<0,001)$. Procedimentos executados por outros profissionais de nível superior representaram $9 \%$ do total e foram mais observados nas UBS do PSF $(p<0,001)$. A proporção de procedimentos de imunização foi de $7 \%$, tendo sido significativamente mais frequente em UBS Tradicionais. Os atendimentos odontológicos também representaram 7\% dos registros e, independente da classificação (preventivos ou dentística / exodontia), não mostraram diferenças estatisticamente significativas entre os modelos de atenção.

A análise dos atendimentos conforme a equipe demonstrou que $43 \%$ foram realizados por profissionais de nível superior e $35 \%$ por profissionais de nível médio, sendo ambas as proporções significativamente maiores nas UBS Tradicionais $(\mathrm{p}<0,001)$. Os atendimentos dos ACS representaram $22 \%$ do total e foram quatro vezes mais frequentes em UBS do PSF $(\mathrm{p}<0,001)$. Ao se examinar a participação apenas dos profissionais com escolaridade superior, observou-se que no PSF os atendimentos de enfermeiros e odontólogos foram cerca de duas vezes maiores que em UBS Tradicionais $(\mathrm{p}<0,001)$. Nestas UBS, os atendimentos de médicos e de outros profissionais de nível superior foram, respectivamente, $40 \%$ e duas vezes maiores que em UBS com PSF $(\mathrm{p}<0,001)$.

As diferenças encontradas segundo o modelo de atenção orientaram análises estratificadas por região (Sul e Nordeste) em cada modelo (Tradicional e Saúde da Família) e a Tabela 2 apresenta os resultados destas análises. 
Tabela 1. Características da demanda atendida em unidades básicas de saúde de acordo com o modelo de atenção. Estudo de Linha de Base, Projeto de Expansão e Consolidação da Saúde da Família,

Universidade Federal de Pelotas, 2008.

\begin{tabular}{|c|c|c|c|c|}
\hline & PSF \% & Tradicional \% & Total \% (n) & $\mathrm{p}$ \\
\hline \multicolumn{5}{|l|}{ Subgrupos $(n=25.901)$} \\
\hline Menores de 5 anos & 14,8 & 14,9 & $14,8(3.836)$ & 0,747 \\
\hline De 5 a 14 anos & 9,8 & 10,9 & $10,1(2.624)$ & 0,006 \\
\hline Mulheres de 15 a 49 anos & 37,9 & 30,8 & $35,6(9.216)$ & 0,000 \\
\hline Homens de 15 a 49 anos & 8,7 & 10,1 & $9,2(2.381)$ & 0,000 \\
\hline De 50 a 64 anos & 16,4 & 18,7 & $17,2(4.445)$ & 0,000 \\
\hline De 65 anos e mais & 12,4 & 14,5 & $13,1(3.399)$ & 0,000 \\
\hline \multicolumn{5}{|l|}{ Escolaridade $(n=23.252)$} \\
\hline Analfabetos & 12,9 & 11,9 & $12,6(2.928)$ & 0,033 \\
\hline Só sabe assinar & 14,6 & 11,8 & $13,7(3.193)$ & 0,000 \\
\hline Fundamental incompleto & 36,1 & 36,8 & $36,3(8.447)$ & 0,367 \\
\hline Fund. comp e médio incompleto & 14,3 & 15,4 & $14,6(3.400)$ & 0,027 \\
\hline Médio completo e superior & 9,9 & 10,2 & $10,0(2.318)$ & 0,517 \\
\hline Fora da idade escolar & 12,2 & 14,0 & $12,8(2.966)$ & 0,000 \\
\hline \multicolumn{5}{|l|}{ Procedimentos $(\mathrm{n}=25.897)$} \\
\hline Imunização & 6,1 & 8,1 & $6,7(1.746)$ & 0,000 \\
\hline Atendimento básico de enfermagem & 30,5 & 36,6 & $32,5(8.415)$ & 0,000 \\
\hline Visita domiciliar & 29,7 & 7,5 & $22,4(5.797)$ & 0,000 \\
\hline Consulta médica & 16,5 & 33,3 & $22,0(5.700)$ & 0,000 \\
\hline Prevenção em odontologia & 4,6 & 4,2 & $4,5(1.161)$ & 0,162 \\
\hline Dentística/exodontia & 2,8 & 2,6 & 2,7 (707) & 0,264 \\
\hline Outro procedimento nível superior & 9,9 & 7,6 & $9,2(2.371)$ & 0,000 \\
\hline \multicolumn{5}{|l|}{ Profissionais $(\mathrm{n}=25.938)$} \\
\hline Nível superior & 37,4 & 53,3 & $42,6(11.059)$ & 0,000 \\
\hline Nível médio & 33,1 & 39,3 & $35,1(9.108)$ & 0,000 \\
\hline Agentes Comunitários de Saúde & 29,5 & 7,5 & $22,2(5.771)$ & 0,000 \\
\hline \multicolumn{5}{|l|}{ Profissionais de nível superior $(\mathrm{n}=11.059)$} \\
\hline Enfermeiro & 31,6 & 15,5 & $24,9(2.757)$ & 0,000 \\
\hline Médico & 44,5 & 63,9 & $52,5(5.808)$ & 0,000 \\
\hline Odontólogo & 20,3 & 12,0 & $16,9(1.867)$ & 0,000 \\
\hline Outros & 3,6 & 8,6 & $5,7(627)$ & 0,000 \\
\hline Total & 100,0 & 100,0 & 100,0 & - \\
\hline
\end{tabular}

Nas UBS com PSF, no Nordeste foram atendidas em maior proporção mulheres de 15 a 49 anos e crianças menores de cinco anos $(\mathrm{p}<0,001)$, além de mais usuários nos extremos de escolaridade $(\mathrm{p}<0,01)$, em comparação com o Sul. Também foram maiores as proporções de visitas domiciliares $(p<0,001)$ e procedimentos em dentística / exodontia $(\mathrm{p}<0,05)$. Os enfermeiros e os odontólogos foram os profissionais que mais responderam pelos atendimentos $(\mathrm{p}<0,05)$.

Nas UBS do PSF do Sul foram significativamente mais frequentes os atendimentos de homens entre 15 e 49 anos, pessoas com 50 anos e mais $(\mathrm{p}<0,001)$ e com ensino fundamental incompleto $(p<0,001)$. Também foram registrados maior número de procedimentos de imuni- zação $(p<0,01)$, de atendimentos básicos de enfermagem e de consultas médicas $(\mathrm{p}<0,001)$. Predominaram os atendimentos de profissionais de nível médio $(\mathrm{p}<0,001)$. Entre os profissionais de nível superior, predominaram os atendimentos médicos $(p<0,001)$.

Em UBS Tradicionais do Nordeste (Tabela 2) foram atendidas mais crianças até cinco anos e mulheres entre 15 e 49 anos $(\mathrm{p}<0,05)$. Em relação à escolaridade, no Nordeste foram registrados mais atendimentos de pessoas com as mais baixas $(\mathrm{p}<0,05)$ e as mais altas escolaridades $(\mathrm{p}<0,001)$. Quanto aos procedimentos, realizaram mais imunização, visitas domiciliares, atendimentos odontológicos e outros de nível superior $(\mathrm{p}<0,05)$. Os ACS atenderam mais no Nor- 
Tabela 2. Características da demanda atendida em unidades básicas de Saúde da Família e Tradicionais, de acordo com a região. Estudo de Linha de Base, Projeto de Expansão e Consolidação da Saúde da Família, Universidade Federal de Pelotas, 2008.

\begin{tabular}{|c|c|c|c|c|c|c|c|c|}
\hline & \multicolumn{4}{|c|}{ Unidades com PSF } & \multicolumn{4}{|c|}{ Unidades Tradicionais } \\
\hline & Sul \% & Nordeste \% & Total \% (n) & p & Sul \% & Nordeste \% & Total \% (n) & $\mathbf{p}$ \\
\hline \multicolumn{9}{|l|}{ Subgrupos } \\
\hline Menores de 5 anos & 12,5 & 16,7 & $14,8(2.557)$ & 0,000 & 13,1 & 18,1 & $14,9(1.279)$ & 0,000 \\
\hline De 5 a 14 anos & 10,2 & 9,4 & $9,8(1.692)$ & 0,084 & 10,3 & 11,9 & $10,9(932)$ & 0,022 \\
\hline Mulheres de 15 a 49 anos & 34,2 & 41,2 & $37,9(6.573)$ & 0,000 & 28,8 & 34,3 & $30,8(2.643)$ & 0,000 \\
\hline Homens de 15 a 49 anos & 10,0 & 7,7 & $8,7(1.515)$ & 0,000 & 10,1 & 10,0 & $10,1(866)$ & 0,873 \\
\hline De 50 a 64 anos & 19,2 & 13,9 & $16,4(2.838)$ & 0,000 & 21,5 & 14,0 & $18,7(1.607)$ & 0,000 \\
\hline De 65 anos e mais & 13,9 & 11,1 & $12,4(2.152)$ & 0,000 & 16,2 & 11,7 & $14,5(1.247)$ & 0,000 \\
\hline \multicolumn{9}{|l|}{ Escolaridade } \\
\hline Analfabetos & 11,0 & 14,6 & $12,9(2.052)$ & 0,000 & 11,2 & 13,2 & $11,9(876)$ & 0,015 \\
\hline Só sabe assinar & 13,7 & 15,4 & $14,6(2.322)$ & 0,003 & 11,2 & 13,1 & $11,8(871)$ & 0,016 \\
\hline Fundamental incompleto & 42,0 & 30,9 & $36,1(5.742)$ & 0,000 & 41,4 & 28,2 & $36,8(2.705)$ & 0,000 \\
\hline Fund. comp e médio incompleto & 13,8 & 14,7 & $14,3(2.268)$ & 0,099 & 15,7 & 14,8 & $15,4(1.132)$ & 0,296 \\
\hline Médio completo e superior & 8,8 & 10,9 & $9,9(1.570)$ & 0,000 & 9,0 & 12,4 & $10,2(748)$ & 0,000 \\
\hline Fora da idade escolar & 10,7 & 15,5 & $12,2(1.938)$ & 0,000 & 11,5 & 18,5 & $14,0(1.028)$ & 0,000 \\
\hline \multicolumn{9}{|l|}{ Procedimentos } \\
\hline Imunização & 6,6 & 5,6 & $6,1(1.056)$ & 0,004 & 7,7 & 8,9 & $8,1(690)$ & 0,047 \\
\hline Atendimento básico de enfermagem & 38,0 & 23,9 & $30,5(5.298)$ & 0,000 & 41,0 & 28,9 & $36,6(3.117)$ & 0,000 \\
\hline Visita domiciliar & 23,1 & 35,4 & $29,7(5.197)$ & 0,000 & 4,1 & 13,6 & $7,5(640)$ & 0,000 \\
\hline Consulta médica & 17,8 & 15,3 & $16,5(2.866)$ & 0,000 & 34,3 & 31,5 & $33,3(2.834)$ & 0,009 \\
\hline Prevenção em odontologia & 4,4 & 4,8 & $4,6(802)$ & 0,238 & 3,9 & 4,8 & $4,2(359)$ & 0,047 \\
\hline Dentística/exodontia & 2,5 & 3,1 & $2,8(489)$ & 0,030 & 2,1 & 3,3 & $2,6(218)$ & 0,000 \\
\hline Outro procedimento nível superior & 7,6 & 12,0 & $9,9(1.722)$ & 0,000 & 7,0 & 8,8 & $7,6(649)$ & 0,002 \\
\hline \multicolumn{9}{|l|}{ Profissionais } \\
\hline Nível superior & 36,9 & 37,8 & $37,4(6.498)$ & 0,228 & 53,6 & 52,7 & $53,3(4.591)$ & 0,469 \\
\hline Nível médio & 40,3 & 26,7 & $33,1(5.744)$ & 0,000 & 42,3 & 33,9 & $39,3(3.364)$ & 0,000 \\
\hline Agentes Comunitários de Saúde & 22,8 & 35,5 & $29,5(5.133)$ & 0,000 & 4,1 & 13,3 & $7,5(638)$ & 0,000 \\
\hline \multicolumn{9}{|l|}{ Profissionais de nível superior } \\
\hline Enfermeiro & 29,5 & 33,3 & $31,6(2.051)$ & 0,001 & 18,1 & 10,8 & $15,5(706)$ & 0,000 \\
\hline Médico & 48,3 & 41,3 & $44,5(2.893)$ & 0,000 & 63,1 & 65,4 & $63,9(2.915)$ & 0,128 \\
\hline Odontólogo & 18,5 & 21,8 & $20,3(1.318)$ & 0,001 & 10,3 & 15,1 & $12,0(549)$ & 0,000 \\
\hline Outros & 3,7 & 3,6 & $3,6(236)$ & 0,856 & 8,5 & 8,7 & $8,6(391)$ & 0,841 \\
\hline Total & 100,0 & 100,0 & 100,0 & - & 100,0 & 100,0 & 100,0 & - \\
\hline
\end{tabular}

deste $(\mathrm{p}<0,001)$ e, entre os de nível superior, os odontólogos realizaram maior proporção de atendimentos $(\mathrm{p}<0,001)$.

Em UBS Tradicionais do Sul foram mais frequentes os atendimentos a usuários com mais idade $(\mathrm{p}<0,001)$ e com ensino fundamental incompleto $(\mathrm{p}<0,001)$. Quanto aos procedimentos, foram realizados mais atendimentos básicos de enfermagem e consultas médicas $(p<0,01)$. Os profissionais de nível médio atenderam mais no Sul e, dos membros da equipe de nível superior, viu-se uma maior participação dos enfermeiros no atendimento $(\mathrm{p}<0,001)$.

\section{Discussão}

A necessidade de avaliações em serviços de saúde, principalmente sob a estratégia saúde da família, vem aumentando e os desafios inerentes aos processos de avaliação já começaram a ser identificados: a saúde-doença como objeto das intervenções, a complexidade das ações e práticas, a subjetividade das avaliações e a necessidade de que seus resultados subsidiem a tomada de decisões $^{3,21,23,29,30}$. Neste estudo, a reduzida proporção de perdas (2\%), a distribuição geográfica e a representatividade das UBS, garantidas pelo processo amostral, juntamente com o tamanho da amostra, permitiram delinear uma importante 
aproximação do perfil da demanda atendida e registrada pelos serviços de atenção básica em um único dia de trabalho nas regiões Sul e Nordeste do Brasil.

Em números absolutos, as unidades que trabalham com a estratégia Saúde da Família tiveram mais atendimentos, decorrente em parte do desenho amostral e da inclusão dos atendimentos realizados por ACS. A amostra da demanda em Atenção Básica à Saúde foi composta em proporções semelhantes de atendimentos realizados na região Sul e NE do Brasil, dois terços dela atendida em serviços do tipo PSF e um terço em serviços do tipo Tradicional.

A distribuição da demanda por grupos populacionais mostrou $24 \%$ de crianças, $37 \%$ de mulheres e $37 \%$ de idosos, sendo esta informação útil para dimensionar a carga de trabalho na atenção básica, caracterizada por ações de cunho preventivo e curativo. Este perfil esteve bastante de acordo com os descritos em outros estudos sobre utilização de serviços básicos de saú$\mathrm{de}^{9,13,22,31}$ e pode refletir também o perfil demográfico e de necessidades, pois varia em função da região e do modelo A demanda constituída predominantemente por imunizações, procedimentos básicos de enfermagem, consultas médicas, visitas domiciliares e procedimentos odontológicos pode revelar as unidades de produção de serviços mais utilizadas dentre a oferta que o processo de trabalho em Atenção Básica disponibiliza à população ${ }^{18}$.

Outro achado importante é o de que apenas $10 \%$ das pessoas atendidas apresentavam o ensino médio completo ou superior, sendo que um quarto das pessoas eram analfabetos ou só sabiam assinar o nome. Este perfil, além de indicar a situação socioeconômica da população que utiliza os serviços da atenção básica, caracteriza as diferenças de contexto entre o sul e o nordeste do país. Esta composição também sugere um viés de utilização em favor dos mais pobres, uma vez que indivíduos de maior escolaridade da área de abrangência dos serviços estejam buscando e utilizando outros serviços. Estes achados são similares aos descritos por Fernandes et al, ao estudarem a utilização de serviços de saúde em áreas cobertas pelo PSF em Porto Alegre ${ }^{32}$.

A distribuição dos atendimentos entre profissionais do nível básico, do nível médio e superior identificou a composição da força de trabalho, com destaque para a participação central de profissionais de enfermagem e de médicos. Logo, considerando que a atenção básica deve realizar ações de promoção da saúde, prevenção e cuida- dos de agravos, é urgente o redimensionamento da equipe de saúde com a inclusão de novos profissionais, com capacidade resolutiva. Neste caso, cabe destacar a participação crescente de odontólogos nos atendimentos em unidades de Saúde da Família, Igualmente será essencial a viabilização dos Núcleos de Apoio à Saúde da Família (NASF) e de seu apoio matricial ao atendimento da demanda de serviços do PSF.

A estratificação das variáveis de acordo com o modelo de atenção mostrou, na Saúde da Família, uma demanda composta predominante de mulheres e crianças, um processo de trabalho desenvolvido por equipe formada por profissionais de diversos níveis de formação e núcleos de origem no campo da saúde, realizando visita domiciliar em uma frequência quatro vezes maior do que o modelo Tradicional. Este, esteve caracterizado pelo predomínio de atendimentos a adultos e idosos através de atividade centrada no médico, embora execute significativamente mais procedimentos básicos de enfermagem do que a SF.

Os profissionais de nível superior, médio e ACS representam, cada um, um terço da força de trabalho na SF, enquanto no modelo tradicional os profissionais de nível superior ultrapassam a metade da força de trabalho. A análise destacada dos profissionais de nível superior mostra que na ESF os enfermeiros e odontólogos representam o dobro da carga de trabalho quando comparada com o modelo tradicional, enquanto o trabalho médico representa $64 \%$ da carga de trabalho nas unidades tradicionais e $45 \%$ nas unidades da ESF.

O perfil da demanda nesta amostra refletiu de forma inequívoca as diferenças entre os modelos de atenção em curso no país. De acordo com Goldbaum ${ }^{18}$, sob o modelo de saúde da família, a organização da assistência e os tipos de profissionais apresentam diferenças, em comparação com a atenção básica "tradicional", que podem modificar os padrões de utilização ou procura de serviços de saúde.

Ao registrar mais atendimentos de pessoas com menor escolaridade, as UBS do PSF podem estar contribuindo para a equidade em saúde ${ }^{3,23}$. Ao registrar mais atendimentos de mulheres em idade reprodutiva e de profissionais não médicos, as UBS do PSF podem estar contribuindo para maiores coberturas de ações de promoção da saúde e prevenção aos agravos.

Apesar dos atendimentos ainda estarem fortemente centrados no profissional médico, é possível evidenciar uma maior participação dos enfermeiros e odontólogos, principalmente no PSF. 
Este fato pode ser devido ao maior número de profissionais médicos por UBS no modelo Tradicional, mas por outro lado pode significar que no PSF há uma maior participação dos demais membros da equipe.

A estratificação das variáveis que descrevem a demanda por região (Sul e Nordeste) de onde se originou, refletiu a estrutura demográfica própria de cada uma das regiões, apontando o predomínio de mulheres e de crianças no NE, assim como de adultos e idosos no Sul 7 . A maior prevalência de visitas domiciliares e de atendimentos por ACS no NE indica a predominância da SF nesta região, enquanto na região Sul foram mais frequentes os atendimentos médicos e os procedimentos básicos de enfermagem, características do modelo Tradicional de organização do processo de trabalho que ainda é hegemônico no Sul do Brasil.

A utilização de um instrumento similar ao documento rotineiramente preenchido pelas equipes $^{33}$ foi um aliado na logística do estudo, pois os campos já eram do conhecimento dos profissionais. Entretanto, importantes informações não puderam ser aproveitadas, dada a elevada proporção de ausência de registro nos documentos. São exemplos desta lacuna os tipos de encaminhamento, os tipos de atendimento e de programa de saúde. Uma das possíveis explicações é a preocupação dos profissionais em preencher apenas os campos exigidos pela gestão para geração de informação unicamente contábil. Outros campos não são devidamente preenchidos na rotina diária dos serviços e, portanto, não podem ser utilizados pelos gestores para a geração de informações sobre a rede dos cuidados de saúde. A proporção de atendimentos que necessitaram de referência a outros níveis de complexidade do sistema poderia ser utilizada como indicador da capacidade resolutiva dos serviços. Camargo Jr et al também apontam as deficiências nas estruturas de informação e comunicação da atenção básica como uma "dificuldade generalizada" em estudo com municípios do mesmo porte em Minas Gerais e no Espírito Santo ${ }^{34}$.

Outro exemplo diz respeito ao tipo de programa de saúde. Mesmo que este campo não se aplique a muitos atendimentos, seu preenchimento adequado para consultas de pré-natal, puericultura e pacientes crônicos possibilitaria numeradores precisos para cálculo de coberturas destes programas.

As equipes, ao identificarem que os registros não são utilizados para a gestão, não se preocupam com o preenchimento de todos os cam- $\operatorname{pos}^{18}$. Este círculo só poderá ser rompido com a qualificação na geração e no uso dos dados ${ }^{35}$, envolvendo necessidades de normatização e padronização de registros, capacitação de recursos humanos e investimentos em infraestrutura, particularmente em tecnologia da informação. Devese destacar aqui a utilidade do aplicativo PACOTAPS $^{27}$, ferramenta desenvolvida para dar conta desta lacuna e distribuída gratuitamente para todas as UBS estudadas.

Para as atividades de prevenção e controle em saúde, a utilização da CID-10 - centrada em doenças - não é específica e os atendimentos seriam melhor classificados através das ações programáticas. Estudando a demanda ambulatorial em três UBS no Rio de Janeiro, Carvalho e colaboradores concluíram que a qualidade dos registros da consulta em prontuário foi precária, faltando importantes informações e os diagnósticos mais frequentes foram classificados como afecções mal-definidas ${ }^{36}$.

Há um consenso sobre a importância central da informação, oriunda de dados de qualidade, não só nos serviços de atenção básica à saúde. $\mathrm{O}$ preenchimento incorreto e insuficiente da declaração de óbito e dos registros hospitalares inviabiliza a produção de informação sobre iniquidades em saúde. Os dados de renda e situação ocupacional na maioria das vezes não são levantados, obstaculizando o estudo mais rigoroso do impacto destas variáveis sociais no perfil de saúde da população $0^{37}$.

Uma das limitações do estudo refere-se à falta de informação sobre a proporção de profissionais que aderiram ao preenchimento dos formulários. Entretanto, como a coleta foi precedida de esclarecimentos e motivação para o estudo em oficina de capacitação com representantes de todas as $\mathrm{UBS}^{26}$, acredita-se que esta lacuna não seja capaz de interferir nos resultados, uma vez que a amostra teve tamanho suficiente para os objetivos desta análise.

Assim, o perfil da demanda, sua estratificação por modelo e por região, além de oferecer informações importantes sobre as características demográficas e de escolaridade dos usuários, pode ser útil no dimensionamento da carga de trabalho nas UBS e na estimativa da necessidade de recursos para atender a esta demanda. Constitui-se, desta forma, em um recurso fundamental para a gestão da Atenção Básica e a organização do cuidado neste nível, ao revelar as vantagens e limitações dos dois modelos de atenção. É necessário que sejam desenvolvidas alternativas criativas para superar as limitações e se aproximar de 
um modelo mais efetivo e eficaz, adequado às características da população de cada região do Brasil e à capacidade de oferta do sistema em um contexto determinado.

Também se espera estar contribuindo para a formação de profissionais de saúde, conscientes das especificidades do processo de trabalho em Atenção Básica, assim como da importância de gerar informações de qualidade para o planejamento, monitoramento e avaliação dos serviços de saúde prestados à população no âmbito do SUS.

\section{Colaboradores}

E Tomasi, LA Facchini e RX Piccini participaram da concepção, delineamento, análise e interpretação dos dados; A Osorio trabalhou na elaboração dos instrumentos e no processamento dos dados, DS Silveira e FV Siqueira participaram da análise e interpretação dos dados e VA Teixeira, AS Dilélio e MFS Maia participaram da revisão de literatura, da redação e revisão do artigo.

\section{Agradecimentos}

Os autores agradecem aos trabalhadores das unidades básicas de saúde pela acolhida e apoio prestado e aos auxiliares de pesquisa, pelo empenho nas diferentes etapas do trabalho de campo. O estudo foi financiado pelo Ministério da Saúde e Banco Mundial no âmbito do Componente 3 do Projeto de Expansão e Consolidação da Saúde da Família

\section{Referências}

1. Travassos C, Martins M. Uma revisão sobre os conceitos de acesso e utilização de servicos de saúde. Cad Saude Publica 2004; 20(Supl. 2):S190-S198.

2. Castro MSM, Travassos C, Carvalho MS. Efeito da oferta de serviços de saúde no uso de internações hospitalares no Brasil. Rev Saude Publica 2005; 39(2): 277-284.

3. Facchini LA, Piccini RX, Tomasi E, Thumé E, Teixeira VA, Silveira DSd, Maia MFS, Siqueira FV, Rodrigues MA, PanizVV, Osório A. Avaliação de efetividade da atenção básica à saúde em municípios das regiões Sul e Nordeste do Brasil: contribuições metodológicas. Cad Saude Publica 2008; 24(Supl. 1):s159s172.

4. Porta M. A dictionaryofepidemiology. $5^{\text {a }}$ ed. New York: Oxford University Press; 2008.

5. Chi C. An event count model for studying health services utilization. Medical Care 1998; 36(12):16391659.

6. Green LA, Fryer GE, Jr., Yawn BP, Lanier D, Dovey SM. The ecology of medical care revisited. $N$ Engl J Med 2001; 344(26):2021-2025.

7. Instituto Brasileiro de Geografia e Estatística (IBGE). PNAD - Pesquisa Nacional por Amostra de Domicílios: sintese de indicadores 2003. Rio de Janeiro: IBGE; 2003.

8. Pinheiro RS, Viacava F, Travassos C, Brito AS. Gênero, morbidade, acesso e utilização de serviços de saúde no Brasil. Cien Saude Colet 2002; 7(4):687707.

9. Mendoza-Sassi R, Béria JU. Utilización de los servicios de salud: una revisión sistemática sobre los factores relacionados. Cad Saude Publica 2001; 17(4):819-32.

10. Sawyer DO, Leite IdC, Alexandrino R. Perfis de utilização de serviços de saúde no Brasil. Cien Saude Colet 2002; 7(4):757-776.

11. Dias da Costa JS, Facchini LA. Utilização de serviços ambulatoriais em Pelotas: onde a população consulta e com que frequência. Rev Saude Publica 1997; 31(4):360-369. 
12. Mendoza-Sassi R, Bria JU, Barros AJD. Outpatient health service utilization and associated factors: a population-based study. Rev Saude Publica 2003; 37(3):372-378.

13. Capilheira MF, da Silva Dos Santos I. Fatores individuais associados a utilização de consultas médicas por adultos. Rev Saude Publica 2006; 40(3):436-443.

14. Barros FC, Victora CG. Epidemiologia da saúde infantil: um manual para diagnósticos comunitários. São Paulo: Hucitec; 1991.

15. Escuder MML, Silva NN, Pereira JCR, Puccini RF, Herrmann AA. Assessing morbidity in the paediatric community. Rev Saude Publica 1999; 33(4):349-357.

16. Schraiber LB, Mendes-Gonçalves RB. Necessidade de saúde e atenção primária. In: Schraiber LB, Nemes MIB, Mendes-Gonçalves RB, organizadores. Saúde do adulto: programa e ações na unidade básica São Paulo: Hucitec; 2000. p. 29-47.

17. World Health Organization (WHO). United Children's Found (UNICEF). Health for all. Geneva: World Health Organization; 1978.

18. Bodstein R. Atenção básica na agenda da saúde. Cien Saude Colet 2002; 7(3):401-423.

19. Brasil. Ministério da Saúde (MS). Saúde da família: uma estratégia de organização dos serviços de saúde. Brasília: Secretaria de Assistência à Saúde; 1996.

20. Conill EM. Ensaio histórico-conceitual sobre a atenção primária à saúde: desafios para a organização de serviços básicos e da estratégia saúde da família em centros urbanos no Brasil. Cad Saude Publica 2008; 24(Supl. 1):S7-S16.

21. Trad LAB, Bastos ACdSB. O impacto sócio-cultural do Programa de Saúde da Família (PSF): uma proposta de avaliação. Cad Saude Publica 1998; 14(2): 429-435.

22. Goldbaum M, Gianini RJ, Novaes HMD, César CLG. Utilização de serviços de saúde em áreas cobertas pelo programa saúde da família (Qualis) no Município de São Paulo. Rev Saude Publica 2005; 39(1):9099.

23. Facchini LA, Piccini RX, Tomasi E, Thumé E, Silveira DS, Siqueira FV, Rodrigues MA. Desempenho do PSF no Sul e no Nordeste do Brasil: avaliação institucional e epidemiológica da Atenção Básica à Saúde. Cien Saude Colet 2006; 11(3):669-681.

24. World Health Organization (WHO). The world health report 2008: primary health care now more than ever. Geneva: World Health Organization; 2008.

25. Brasil. Ministério da Saúde (MS). Termo de referência para o estudo de linha de base nos municípios selecionados para o componente 1 do PROESF. Brasília: Ministério da Saúde (MS); 2004.

26. Piccini RX, Facchini LA, Tomasi E, Thumé E, Silveira DS, Teixeira VA, Maia MFS, Osório A, Siqueira FCV, Dilélio AS. Capacitação no PROESF-UFPel: contribuições à educação de trabalhadores e à pesquisa em atenção básica à saúde nas regiões Sul e Nordeste do Brasil. In: Hartz Z, Felisberto E, Silva LV, organizadores. Meta-avaliação da atenção básica em saúde: teoria e prática. Rio de Janeiro: Fiocruz; 2008. p. 167-197.
27. Tomasi E, Facchini LA, Osorio A, Fassa AG. Aplicativo para sistematizar informações no planejamento de ações de saúde pública. Rev Saude Publica 2003; 37(6):800-806.

28. Facchini LA, Piccini RX, Tomasi E, Thumé E, Silveira DS. Relatório Final do Projeto de Monitoramento e Avaliação do Programa de Expansão e Consolidação do Saúde da Família (PROESF). Pelotas, RS: UFPEL; 2006.

29. Novaes HM. Avaliação de programas, serviços e tecnologias em saúde. Rev Saude Publica 1999; 34(5):547-559.

30. Carvalho AI, Bodstein RC, Hartz Z. Concepções e abordagens na avaliação em promoção da saúde. Cien Saude Coletiva 2004; 9(3):532-533.

31. Travassos C, Viacava F, Pinheiro R, Brito A. Utilização dos serviços de saúde no Brasil: gênero, características familiares e condição social. Rev $\mathrm{Pa}$ nam Salud Publica 2002; 11(5-6):365-373.

32. Fernandes LCL, Bertoldi AD, Barros AJD. Utilização dos serviços de saúde pela população coberta pela Estratégia de Saúde da Família. Rev Saude Publica 2009; 43(4):595-603.

33. Sistema de Informações Ambulatoriais do SUS 2008 [database on the Internet]. [acessado 2008 out 17]. Disponível em: http://w3.datasus.gov.br/siasih/ siasih.php.2008.

34. Camargo Jr. KRd, Campos EMS, BustamanteTeixeira MT, Mascarenhas MTM, Mauad NM, Franco TB, Ribeiro LC, Alves MJM. Avaliação da atenção básica pela ótica políticoinstitucional e da organização da atenção com ênfase na integralidade. Cad Saude Publica 2008; 24(Sup. 1):S58-S68.

35. Viacava F, Informações em saúde: a importância dos inquéritos populacionais. Cien Saude Coletiva [periódico na Internet]. 2002 [acessado 2008 jun 06]; 7(4):[607-621]. Disponível em: bvsms.saude.gov.br/ bvs/is_digital/is_0203/pdfs/IS23(2)040.pdf

36. Carvalho MS, D'Orsi E, Prates EC, Toschi WDM, Shiraiwa T, Campos TP, Ell E, Garcia NL, Junqueira AP, Serräo SA, Tavares EL. Demanda Ambulatorial em Três Serviços da Rede Pública do Município do Rio de Janeiro, Brasil. Cad Saude Publica 1994; 10(1): 17-29.

37. Magalhães R. Monitoramento das desigualdades sociais em saúde: significados e potencialidades das fontes de informação. Cien Saude Colet 2007; 12(3): 667-673.

Artigo apresentado em 01/04/2009

Aprovado em 22/10/2009

Versão final apresentada em 17/11/2009 